\title{
Development and Evaluation of Alginate Membranes with Curcumin-Loaded Nanoparticles for Potential Wound-Healing Applications
}

\author{
Mónica C. Guadarrama-Acevedo ${ }^{1,+}$, Raisa A. Mendoza-Flores ${ }^{1, \dagger}$, María L. Del Prado-Audelo ${ }^{1,2}$, \\ Zaida Urbán-Morlán ${ }^{1}$, David M. Giraldo-Gomez ${ }^{3}{ }^{\circledR}$, Jonathan J. Magaña ${ }^{4}$, \\ Maykel González-Torres 5,6 (D), Octavio D. Reyes-Hernández ${ }^{7}$, Gabriela Figueroa-González ${ }^{8}$, \\ Isaac H. Caballero-Florán ${ }^{1,9}$, Carla D. Florán-Hernández ${ }^{4}$, Benjamín Florán ${ }^{9} \mathbb{D}$, \\ Hernán Cortés ${ }^{4}$ (D) and Gerardo Leyva-Gómez ${ }^{1, *(\mathbb{D})}$ \\ 1 Departamento de Farmacia, Facultad de Química, Universidad Nacional Autónoma de México, Ciudad \\ Universitaria, Circuito Exterior S/N, Del. Coyoacán, Ciudad de México 04510, México \\ 2 Laboratorio de Posgrado en Tecnología Farmacéutica, FES-Cuautitlán, Universidad Nacional Autónoma de \\ México, Cuautitlán Izcalli 54740, México \\ 3 Departamento de Biología Celular y Tisular, Facultad de Medicina, Universidad Nacional Autónoma de \\ México (UNAM), Edificio "A" 3er piso, Circuito Interior, Avenida Universidad 3000, Ciudad Universitaria, \\ Coyoacán, Ciudad de México 04510, México \\ 4 Laboratorio de Medicina Genómica, Departamento de Genética, Instituto Nacional de Rehabilitación Luis \\ Guillermo Ibarra Ibarra, Ciudad de México 14389, México \\ 5 CONACyT-Laboratorio de Biotecnología, Instituto Nacional de Rehabilitación Luis Guillermo Ibarra Ibarra, \\ Ciudad de México 14389, México \\ 6 Instituto Tecnológico y de Estudios Superiores de Monterrey, Campus Ciudad de México 14380, México \\ 7 Laboratorio de Biología Molecular del Cáncer, UMIEZ, Facultad de Estudios Superiores Zaragoza, \\ Universidad Nacional Autónoma de México, Ciudad de México 09230, México \\ 8 CONACyT-Laboratorio de Genómica, Dirección de Investigación, Instituto Nacional de Cancerología. Av. \\ San Fernando 22, Tlalpan, Sección XVI, Ciudad de México 14080, México \\ 9 Departamento de Fisiología, Biofísica \& Neurociencias, Centro de Investigación y de Estudios Avanzados \\ del Instituto Politécnico Nacional, Ciudad de México 07360, México \\ * Correspondence: gerardoleyva@hotmail.com; Tel.: +52-(55)-5622-3899 (ext. 44408) \\ + Mónica C. Guadarrama-Acevedo and Raisa A. Mendoza-Flores equally contributed as first authors to the \\ present work.
}

Received: 6 June 2019; Accepted: 30 July 2019; Published: 3 August 2019

check for updates

\begin{abstract}
Non-biodegradable materials with a low swelling capacity and which are opaque and occlusive are the main problems associated with the clinical performance of some commercially available wound dressings. In this work, a novel biodegradable wound dressing was developed by means of alginate membrane and polycaprolactone nanoparticles loaded with curcumin for potential use in wound healing. Curcumin was employed as a model drug due to its important properties in wound healing, including antimicrobial, antifungal, and anti-inflammatory effects. To determine the potential use of wound dressing, in vitro, ex vivo, and in vivo studies were carried out. The novel membrane exhibited the diverse functional characteristics required to perform as a substitute for synthetic skin, such as a high capacity for swelling and adherence to the skin, evidence of pores to regulate the loss of transepidermal water, transparency for monitoring the wound, and drug-controlled release by the incorporation of nanoparticles. The incorporation of the nanocarriers aids the drug in permeating into different skin layers, solving the solubility problems of curcumin. The clinical application of this system would cover extensive areas of mixed first- and second-degree wounds, without the need for removal, thus decreasing the patient's discomfort and the risk of altering the formation of the new epithelium.
\end{abstract}


Keywords: wound dressing; polymeric membrane; nanoparticles; curcumin; alginate; pluronic F68; drug skin permeation; Franz cells; tape stripping

\section{Introduction}

Human skin exerts a pivotal function as a protection barrier against diverse exogenous noxious factors; however, it is exposed and undergoes diverse types of injuries, including burns, ulcers, trauma, lacerations, and acute or chronic wounds, which may compromise its integrity [1].

In this regard, when skin is damaged, a specialized and highly regulated dynamic process immediately takes place: wound healing [2]. The main goal of wound healing is to restore tissue integrity and to achieve homeostasis; however, this process may be complicated by distinct intrinsic and extrinsic factors [2]. Thus, in order to accelerate wound healing, a variety of wound dressings have been designed.

Irrespective of the type of wound, the main function of dressings is to aid in the repair of the wound through the reduction of pain and inflammation, to protect the damaged tissue from pathogenic agents, and to enhance cell differentiation and proliferation [3]. Although there several types of dressings which are commercially available, many of these present some drawbacks, such as an inefficient absorption of exudates, poor protection against infections by microbes, the lack of ability to maintain humidity, and the triggering of allergic effects [4]. In addition, several dressings may adhere to the wound and require constant changes, which may interfere with the granulation process and delay the healing course.

Therefore, in recent years, there has been increasing interest in asymmetric membranes as an alternative for designing wound dressings [5]. These types of membranes possess multiple advantages, such as structural similarity with the skin, an ability to absorb exudates due to their porous structure, and improved cell adhesion and proliferation [6,7]. Different polymers have been employed for their development, including chitosan, hyaluronic acid, collagen, poly vinyl alcohol (PVA), polycaprolactone (PCL), and alginate [8]. In particular, alginate is a biopolymer extracted from seaweed and has shown several unique properties, such as biodegradability, good hydrophilicity, and good biocompatibility [4]. Alginate has exhibited potential for improving wound healing due to its hemostatic properties; moreover, it may reduce microbial infections, enhance the absorption of exudates, and decrease allergic reactions [9]. These features render alginate an interesting option for wound dressings. In addition, alginate membranes possess the advantage that they may be functionalized with bioactive compounds that enhance their healing properties.

In this regard, curcumin is a natural compound that possesses a plethora of biological activities, including antimicrobial, antifungal, anti-inflammatory, and antioxidant effects [10-12]. In addition, curcumin improves wound healing, enhances epithelial regeneration, and increases the proliferation of fibroblasts $[13,14]$. Thus, curcumin could be a suitable pharmacological agent for the elaboration of wound dressings. However, curcumin exhibits low bioavailability and is unstable in neutral and alkaline aqueous solutions, as well as in hydrophilic topical preparations [12,15]. These drawbacks could be overcome by a nanoparticle formulation that permits the controlled and gradual release of the compound.

Therefore, the objective of this study was to design and develop a novel wound dressing comprising an alginate membrane and PCL nanoparticles loaded with curcumin and stabilized with Pluronic ${ }^{\circledR}$ F-68 (CNp) for possible application in wound healing. The wound dressing was physicochemically characterized, and in vivo and ex vivo permeation assays were performed. 


\section{Materials and Methods}

\subsection{Materials}

For CNp fabrication, we used PCL (Mn 80,000 g/mol), Pluronic ${ }^{\circledR}$ F-68, and trehalose dihydrate, which were purchased from Sigma-Aldrich ${ }^{\circledR}$ (Merck KGaA, Darmstadt, Germany), whereas methanol, ethyl acetate, and curcumin were supplied by Spectrum ${ }^{\circledR}$ (Spectrum Laboratory Products, CA, USA). For membrane elaboration and characterization, we employed sodium alginate [(SA) (300-700 cps $1.0 \%$, RV, $20 \mathrm{rpm}, 25^{\circ} \mathrm{C}$ ], glycerol (Gly), propylene glycol (Prop), and Tween 80, which were purchased from Droguería Cosmopolita (Mexico City, Mexico). PVA, Pluronic ${ }^{\circledR}$ F-127, acetone, and phosphate buffered saline (PBS) solution were supplied by Sigma-Aldrich ${ }^{\circledR}$ (Merck KGaA, Darmstadt, Germany). Kollidon ${ }^{\circledR} 30$ [Polyvinylpyrrolidone k 30; (PVP k30)] was obtained from BASF (USA). Ethyl acetate was acquired from Distribuidora Química Alvi (State of Mexico, Mexico). Methanol was purchased from Fermont (Nuevo León, Mexico). All other chemicals and reagents were of at least analytical-grade quality.

\subsection{Preparation of PCL Nanoparticles Loaded with Curcumin (CNp)}

$\mathrm{CNp}$ were prepared by the emulsification-diffusion method, as previously described by Quintanar-Guerrero, et al. [16]. Briefly, the organic saturated phase and aqueous saturated phase were obtained by the saturation of an ethyl acetate and distilled water mixture at a 1:1 ratio. After that, 2\% $(w / v)$ solution of PCL was prepared by dissolving $400 \mathrm{mg}$ of PCL in $20 \mathrm{~mL}$ of the organic saturated phase; once PCL was dissolved, $100 \mathrm{mg}$ of curcumin was added. At the same time, $5 \%(w / v)$ of Pluronic ${ }^{\circledR}$ F-68 solution was prepared using an aqueous saturated phase as solvent. In order to obtain an emulsion, both solutions were mixed at a 1:2 ratio with a high-speed homogenizer (Ultra Turrax T18; IKA ${ }^{\circledR}$ ) at $14,000 \mathrm{rpm}$ for $10 \mathrm{~min}$ at room temperature. Then, $160 \mathrm{~mL}$ of water was added to the emulsion to generate polymer aggregation, and the system was maintained under the same conditions for $10 \mathrm{~min}$. The organic solvent was evaporated by a rotary vacuum (Heidolph ${ }^{\circledR}$, Schwabach, Germany), and the nanoparticle suspension obtained was centrifuged at $15,557 \mathrm{~g}$ for $30 \mathrm{~min}$ at $25^{\circ} \mathrm{C}$. Finally, the pellet was dissolved in distilled water. In order to assess the thermal stability and the properties of the nanoparticles, $\mathrm{CNp}$ were frozen and lyophilized at $-49^{\circ} \mathrm{C}, 0.05 \mathrm{mBar}$ for $24 \mathrm{~h}$, employing $5 \%$ w/v trehalose dihydrate as a cryoprotectant.

\subsection{Physicochemical Characterization of $\mathrm{CNp}$}

\subsubsection{Particle Size and Zeta Potential Assessment}

The particle size and distribution (polydispersity index, PDI) of the CNp were evaluated by dynamic light scattering. On the other hand, to determine the Zeta potential of CNp, laser Doppler velocimetry was employed. CNp dispersions were assessed five times at $25^{\circ} \mathrm{C}$ in a Zetasizer (Malvern Instrument ZS90; Malvern, UK).

\subsubsection{Atomic Force Microscopy (AFM)}

$\mathrm{CNp}$ size and geometry were analyzed by atomic force microscopy (AFM) with a scanning probe microscope (JSPM-4210, JEOL ${ }^{\circledR}$, Tokyo, Japan). In brief, the CNp dispersion was obtained after centrifugation, and the pellet resuspension was diluted to 1:100 with distilled water. A drop was placed on a coverslip, allowing it to dry at room temperature. The coverslip with the drop was held in place with carbon tape, and room-temperature conditions were utilized to assess the samples.

\subsubsection{Drug Loading and Entrapment Efficiency of CNp}

To calculate entrapment efficiency (EE) and drug loading (DL), the CNp dispersion was centrifuged at $15,557 \mathrm{~g}$ for $40 \mathrm{~min}$; then, the sediment was resuspended in ethyl acetate and the absorbance was 
measured by UV-Vis spectrophotometry at $420 \mathrm{~nm}\left(\mathrm{DLAB}^{\circledR}{ }^{\circledR}, \mathrm{SP}-\mathrm{UV} 1000\right.$, Beijing, China). The amount of curcumin was obtained by interpolation in a calibration curve $\left(R^{2}\right.$ coefficient $\left.=0.99985\right)$.

The percentages of EE and DL were calculated from the equations below:

$$
\begin{aligned}
& \% \mathrm{EE}=\frac{\mathrm{CN}}{\mathrm{IC}} \times 100 \\
& \% \mathrm{DL}=\frac{\mathrm{CN}}{\mathrm{N}} \times 100
\end{aligned}
$$

where $\mathrm{CN}=$ the amount of curcumin in nanoparticles, IC = the initial amount of curcumin, and $\mathrm{N}=$ the number of nanoparticles.

\subsection{Preparation of Polymer Gels and Membranes}

Four membrane formulations based on SA (M1, M2, M3, and M4) (See Table 1) were prepared using the solvent casting method as published by Karki et al. [17]. First, SA and the polymer were dissolved separately in injectable water by stirring at $35^{\circ} \mathrm{C}$. After dissolution, they were mixed with each other by mechanical stirring. Then, the plasticizer (or a plasticizer mixture) was added by stirring at room temperature until a homogeneous gel was obtained. In order to eliminate bubbles from the gel, it was centrifuged at $636 \mathrm{~g}$ for $20 \mathrm{~min}$ at room temperature (BIOBASE, BKC-TH18II, Shandong, China).

Table 1. Formulation of alginate membranes with different polymers as plasticizers. PVA: poly vinyl alcohol; PVP: polyvinylpyrrolidone; CNp: polycaprolactone (PCL) nanoparticles loaded with curcumin.

\begin{tabular}{cccccc}
\hline \multirow{2}{*}{ Formulation Code } & \multirow{2}{*}{ SA $(\% w / v)$} & \multicolumn{2}{c}{ Polymer } & \multicolumn{2}{c}{ Plasticizer } \\
\cline { 3 - 6 } & & PVA $(\% w / v)$ & PVP $(\% w / v)$ & Gly $(\% w / v)$ & Prop $(\% v / v)$ \\
\hline & 4 & 2 & - & 10 & - \\
M2 & 4 & 2 & - & 10 & 12 \\
M4 & 4 & - & 2 & 10 & - \\
CNp-M4 & 4 & - & 2 & 10 & 12 \\
\hline
\end{tabular}

In order to obtain the membranes, $10 \mathrm{~g}$ of each gel, prepared with the previously mentioned methodology, was poured into a Teflon cast $12 \mathrm{~cm}$ in diameter and left to dry into an oven (OAKTON Stable Temp, IL, USA) at $75.0 \pm 0.5^{\circ} \mathrm{C}$ for $3 \mathrm{~h}$.

\subsubsection{Preparation of Nanoparticle-Coated Alginate Membranes (CNp-M4)}

The methodology described previously was followed to prepare our nanoparticle-coated alginate membrane (CNp-M4), but the vehicle utilized was a dispersion of $\mathrm{CNp}$ to obtain $0.01 \%$ w/v of curcumin instead of injectable water. In order to prepare the membranes, the gel obtained was poured into a Teflon cast and left to dry in an oven at $40.0 \pm 0.5^{\circ} \mathrm{C}$ for $4 \mathrm{~h}$.

\subsection{Physicochemical Characterization of Membranes}

\subsubsection{Swelling Test}

Samples of M1, M2, M3, M4, and CNp-M4 membranes were cut to a size of $1 \times 1 \mathrm{~cm}$ and weighed on pre-weighed aluminum trays. The samples were divided into five blocks, corresponding to different times as follows: 5, 10, 20,30, and $60 \mathrm{~min}$. The membranes on the trays were placed on a flat surface, and $750 \mu \mathrm{L}$ of PBS $1 \mathrm{X}$ pH 7.4 was added to each sample.

Once the established time had elapsed, the trays were turned vertically for 2 min on absorbent paper, allowing the draining and absorbption of the excess of PBS. After that time, the membranes in the trays were weighed again [18]. The assessment was performed in triplicate for each of the different times. 
The swelling percentage (\%S) was calculated using the following formula [19]:

$$
\% \mathrm{~S}=\frac{\mathrm{M}_{\mathrm{S}}-\mathrm{M}_{\mathrm{d}}}{\mathrm{M}_{\mathrm{d}}} \times 100
$$

where $M_{s}$ and $M_{d}$ are the weight of the swollen membrane and dried membrane, respectively.

\subsubsection{Mechanical Test}

Tensile strength (TS) and the elongation at a break (\%E) of M1, M2, M3, M4, and CNp-M4 were determined using a Sintech $\frac{1}{2}$ testing machine (MTS, USA), which was equipped with a 100-N load cell at a crosshead speed of $2.4 \mathrm{~mm} \mathrm{~s}^{-1}$. Three samples of each formulation were cut into a dumbbell shape with a width of $10 \mathrm{~mm}$ and an effective length of $40 \mathrm{~mm}$ between the clamps at the beginning of the measurement. The thickness of each sample was measured using a Vernier at five different points before testing, and the average of these was employed for TS calculation. The load (Lb) and displacement $(\mathrm{mm})$ of each film were recorded during the stretching. TS and $\% \mathrm{E}$ were calculated by Equations (4) and (5), as published by Karki et al. [17]:

$$
\begin{gathered}
\mathrm{TS}=\frac{\mathrm{F}}{\mathrm{A}} \\
\% \mathrm{E}=\frac{\mathrm{D}}{\mathrm{L}} \times 100
\end{gathered}
$$

where TS is reported in MPa, $\mathrm{F}$ is the maximum load $(\mathrm{N})$ required to break the film, and $\mathrm{A}$ is the initial cross-sectional area in $\mathrm{mm}^{2}$. In Equation (5), $\mathrm{D}$ is the displacement of the film elongation at the rupture and $\mathrm{L}$ is the initial length.

\subsubsection{Thermogravimetric Analysis (TGA)}

The thermal stability of the membranes (M4, CNp-M4), CNp, and curcumin was evaluated through TGA, employing a Hi-Res TGA 2950 Thermogravimetric Analyzer (Modulated TA Instruments, New Castle, DE, USA). In brief, $5 \mathrm{mg}$ of each sample was analyzed starting at room temperature and increasing to $500{ }^{\circ} \mathrm{C}$ at a heating rate of $10^{\circ} \mathrm{C} /$ min under a nitrogen atmosphere.

\subsubsection{Differential Scanning Calorimetry (DSC)}

The thermal properties of the membranes (M4, CNp-M4), CNp, and curcumin were determined with the DSC 2910 (Modulated TA Instruments, DE, USA). Lyophilized samples were placed in hermetic aluminum cells and evaluated starting at room temperature and increasing to $250{ }^{\circ} \mathrm{C}$ at a heating rate of $10^{\circ} \mathrm{C} / \mathrm{min}$ under a nitrogen atmosphere.

\subsection{5. $\mathrm{pH}$ Values}

The $\mathrm{pH}$ values of M1, M2, M3, M4, and CNp-M4 polymer gels was measured through a $\mathrm{pH}$ meter. The samples were put into contact with the electrode until a constant value was obtained. The $\mathrm{pH}$ meter was previously calibrated against standard solutions to ensure the highest level of accuracy.

\subsubsection{Structure and Morphology of M4 and CNp-M4 Membranes}

In order to analyze the structure and morphology of the membranes, samples were cut in a circular shape $1 \mathrm{~cm}$ in diameter and in a longitudinal section and were analyzed using a scanning electron microscope (Jeol-JCM 6000, MA, USA) at 100× and 220× magnification; then, photographs were taken in four different fields.

The pore diameter and membrane width were measured using a software package (ImageJ, MD, USA), and the average was calculated. Pore number was counted field-by-field, and the average was determined. 
In order to evaluate the transparency of M4 and CNp-M4 membranes, samples were cut into a circular shape $3 \mathrm{~cm}$ in diameter and these were placed over an image (before swelling); then, they were observed and photographed. Afterward, $1 \mathrm{~mL}$ of PBS $1 \mathrm{X}$ was added to the samples, and after $20 \mathrm{~min}$ (swelling process), photographs were taken of the samples.

\subsubsection{In Vitro Release Study of Drug Dispersion, CNp and CNp-M4 Membrane}

To evaluate the curcumin release profile from the CNp and CNp-M4 membrane, the direct dispersion method was applied. For the drug and $\mathrm{CNp}$ dispersion, a certain amount of curcumin (and the equivalent in nanoparticles) was dissolved into PBS solution (pH 7.4, Pluronic ${ }^{\circledR}$ F-127 2\% $w / v)$, divided into sets of three tubes each, and placed in a shaker incubator, maintaining this at $37^{\circ} \mathrm{C}$. For the CNp-M4 membrane, disks $1 \mathrm{~cm}$ in diameter were placed in tubes with the PBS solution under the conditions previously described. At the defined times of $0,0.25,0.5,1,2,4,6,24$, and $48 \mathrm{~h}$, one set of tubes was removed from shaking and centrifuged at $18,514 \mathrm{~g}$ for $30 \mathrm{~min}$. The curcumin released in the supernatant was quantified by UV-Vis spectrophotometry at $420 \mathrm{~nm}$.

The results of the release tests of $\mathrm{CNp}$ and $\mathrm{CNp}-\mathrm{M} 4$ membrane were analyzed by mathematical models such as zero-order, first-order, the Higuchi model, and the Korsmeyer-Peppas model to predict the drug release mechanism.

\subsection{Permeation Assays}

\subsubsection{Ex Vivo Permeation Assay}

Porcine skin was obtained from the back of pig ears within $12 \mathrm{~h}$ after slaughter. The pig ears were cut into circular sections $3 \mathrm{~cm}$ in diameter. The excess of fat was removed with surgical scissors, and the samples were washed with saline solution. The samples were divided into three groups: drug dispersion with $0.01 \% w / v$ of curcumin $(2 \mathrm{~mL} ; n=3), \mathrm{CNp}$ dispersion with $0.01 \%$ w/v of curcumin ( $2 \mathrm{~mL} ; n=3)$, and CNp-M4 membranes ( $3 \mathrm{~cm}$ in diameter; $n=3)$.

The experiments were conducted in 12 independent Franz cells with a diffusion area of $7.07 \mathrm{~cm}^{2}$. The freshly excised skin was placed into Franz cells, and the stratum corneum remained in contact with the donor compartment, with the dermis facing the receptor compartment. This was filled with $30 \mathrm{~mL}$ of $0.1 \mathrm{M}$ PBS solution ( $\mathrm{pH} 7.4$ ) with $2.5 \%$ of Tween 80 and maintained under constant stirring at $400 \mathrm{rpm}$.

Franz cells were immersed in a water bath at a constant temperature of $37.0 \pm 0.5{ }^{\circ} \mathrm{C}$. At predetermined times $(1,2,3,4,5,6,7,8,22,24,26,28$, and $30 \mathrm{~h}), 1000 \mu \mathrm{L}$ of medium was removed from the receptor compartment and replaced with $1000 \mu \mathrm{L}$ of fresh receptor medium.

At the end of the test, the skin samples were carefully removed from the Franz cells to conduct the tape-stripping test, following the in vivo permeation assay methodology (described in Section 2.6.2). All tapes were placed in a flask with $40 \mathrm{~mL}$ of acetone and mechanically stirred for $15 \mathrm{~h}$. Subsequently, each skin sample was fragmented into small pieces using surgical scissors. The curcumin was extracted with $25 \mathrm{~mL}$ of ethyl acetate:methanol at a ratio of 9:1. The extract was centrifuged at 10,174 $\mathrm{g}$ for $10 \mathrm{~min}$. All samples were analyzed by UV-visible spectrophotometry at a wavelength of $420 \mathrm{~nm}$.

\subsubsection{In Vivo Permeation Assay}

To evaluate the in vivo permeation of the CNp-M4 membrane through the stratum corneum, the tape-stripping technique was employed. Four healthy Mexican males aged 22-36 years were recruited as volunteers. The individuals had neither a history of skin disorders nor had used cosmetic products on their forearms $24 \mathrm{~h}$ prior to the test. Written informed consent was obtained from each volunteer before each study.

Four sites $(3 \times 3 \mathrm{~cm})$ were demarcated: two on the right forearm and two on the left forearm. Before administering the treatment, the sites were cleaned with cotton impregnated with water. Three treatments were administered to each volunteer. The CNp dispersion with $0.01 \%$ of curcumin $(2 \mathrm{~mL})$ and the drug dispersion at the same concentration were contained within a glass cylinder on 
the left forearm. The CNp-M4 membrane ( $3 \mathrm{~cm}$ in diameter) was put on the right forearm, and $1.75 \mathrm{~mL}$ of distilled water was added onto the surface of the membrane. The last site on the right forearm was used for the blank. The treatments were in contact with the skin for $6 \mathrm{~h} \mathrm{[20].} \mathrm{Once} \mathrm{the} \mathrm{time} \mathrm{had} \mathrm{elapsed,}$ the membrane was removed with steel nippers. The skin was subjected to 15 successive tape strips $\left(\right.$ Scotch $\left.{ }^{\circledR} 3 \mathrm{M}^{\circledR}\right)$, previously cut into $3 \times 3 \mathrm{~cm}$ squares. In each case, the site was pressed uniformly by sliding a spatula over the surface of the tape five times; then, it was removed by pulling it with steel nippers from the lower right to the upper left end. The blank site was subjected to the same procedure. Each tape was immersed in $15 \mathrm{~mL}$ of acetone in a different amber glass bottle with a lid. All of the bottles were placed under mechanical agitation for 15 h; subsequently, the samples were filtered to remove the glue particles. The quantification of curcumin was performed by UV-visible spectrophotometry at a wavelength of $420 \mathrm{~nm}$. Each sample was analyzed in triplicate.

\section{Results and Discussion}

\subsection{Physicochemical Characterization of $\mathrm{CNp}$}

\subsubsection{Particle Size and Zeta Potential Assessment}

CNps were obtained, and their mean particle size and PDI were $148.3 \pm 1.9 \mathrm{~nm}$ and $0.044 \pm 0.020$, respectively. These values were expected to improve dermal permeation [21], since nanoparticles below $500 \mathrm{~nm}$ have a larger surface area-to-volume ratio, which ensures direct contact with the stratum corneum and skin appendages [6,7]. Moreover, the PDI value was below 0.1, which indicates that the small size measured in the sample is reliable and monodisperse [22].

On the other hand, CNp exhibited a zeta potential value of $-7.32 \pm 0.03 \mathrm{mV}$. In this regard, zeta potential is commonly employed to measure the charge in the nanoparticles and/or electrostatic repulsion [1], and the literature indicates that nanoparticles are stable in suspension with a zeta potential above $\pm 30 \mathrm{mV}$ [23]. Despite the CNp zeta potential value not being in this range, it should be considered that Pluronic ${ }^{\circledR}$ F-68 was added as a stabilizer of nanoparticles, which provides them with stability by means of a repulsion effect through a steric mechanism [24]. Likewise, CNp possesses a negative charge, which is related to the carboxylic end group of PCL [25]; thus, negatively charged nanoparticles could permeate adequately in conjunction with the negative charges existing on the skin [26].

\subsubsection{Atomic Force Microscopy (AFM)}

The morphology and size of CNp were evaluated by AFM. In agreement with the particle-size and zeta-potential assessments, the nanoparticles demonstrated a spherical shape and a size of approximately $200 \mathrm{~nm}$ with no agglomeration (Figure 1) [27]. These results support the idea that the small size of $\mathrm{CNp}$ could improve the dermal permeation of curcumin, which would increase its anti-inflammatory, antimicrobial, and wound-healing activities [6,12]. 


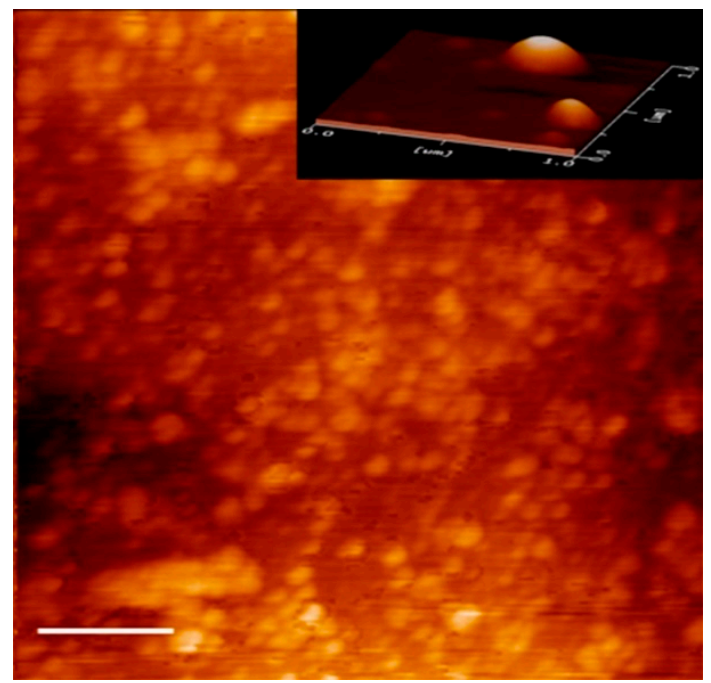

Figure 1. Atomic force microscopy (AFM) topography images of the CNp. The size bar is $1 \mu \mathrm{m}$.

\subsubsection{Drug Loading and Entrapment Efficiency of Nanoparticles}

In order to determine the amount of curcumin entrapped inside the $\mathrm{CNp}$, the DL and EE were determined. The CNp showed DL and EE values of $4.9 \pm 0.7 \%$ and $96.01 \pm 0.95 \%$, respectively. It is known that the emulsification diffusion method ensures high encapsulation efficiencies (generally $>70 \%$ ) [28]. On the other hand, DL depends on nanoparticle structure and methodology [29], whereas both DL and EE depend on the interactions between the drug, the matrix, and the medium [29].

\subsection{Physicochemical and Mechanical Characterization of Nanoparticle-Coated Alginate Membranes as Wound Dressings}

\subsubsection{Swelling Test}

From a practical point of view, the membranes should absorb the exudate from the wound and at the same time provide a moist environment that promotes healing. For this reason, the percentage of swelling of five different formulations was determined by weight difference (Figure 2).

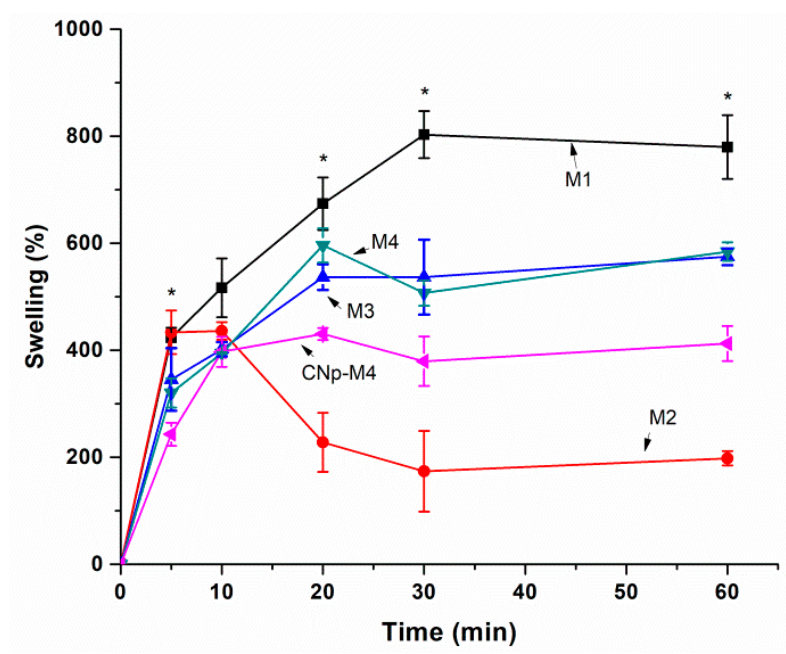

Figure 2. Percentage of swelling in alginate membranes as a function of time. Effect on swelling capacity by the addition of PVA + glycerin (M1), PVA + glycerin + propylene glycol (M2), PVP + glycerin (M3), PVP + glycerin + propylene glycol (M4), and CNp + PVP + glycerin + propylene glycol $(\mathrm{CNp}-\mathrm{M} 4)($ mean $\pm \mathrm{SE} ; n=3)$. There were significant differences when ANOVA was applied at 5, 20, 30 , and $60 \mathrm{~min}(p<0.05)$. 
Figure 2 depicts a difference at a time of $20 \mathrm{~min}$, when propylene glycol is added to the formulation with PVA (M2) in comparison to M1; however, there is no significant difference between using or not using propylene glycol in the formulation combined with PVP (M3 and M4, respectively). In order to determine the differences in the percentage of swelling according to each formulation as a function of time, an ANOVA was performed. It was demonstrated that both the formulation and the time during which the membrane was exposed to PBS exerted a statistically significant effect on swelling with a 95\% confidence level.

The formulation with the highest swelling value was $\mathrm{M} 1$, at $30 \mathrm{~min}(802.8 \pm 76.0 \%)$, while the addition of propylene glycol (M2) decreased the swelling percentage after $10 \mathrm{~min}$. On the other hand, M2 showed the lowest value in the swelling test before dissolving. The addition of propylene glycol to the polyvinyl alcohol mixture could increase the intermolecular interactions between both excipients via hydrogen bonds in the -OH groups, favored by a wide steric disposition, but could decrease the entry capacity of water molecules and their interaction with alginate by saturation, decreasing the swelling ability. In the case of the interaction of propylene glycol and PVP, the situation could be the opposite. The interaction of both excipients is lower, resulting in a lower swelling capacity of the alginate; i.e., the excipient that determines the majority of the response. This phenomenon is confirmed with the profile observed for CNp-M4 in relation to M4.

Namely, when water enters the polymer matrix, the chains begin to relax, giving rise to the opening of the polymer networks. This promotes the penetration of more water; however, in the last stages of swelling, the diffusion coefficient is diminished because the chains are completely relaxed and near equilibrium [30].

The membranes began to dissolve after being exposed to PBS for a longer period of time. According to the composition of the medium, polymers undergo degradation and erosion processes. When a polymer degrades, the chains are cleaved into oligomers and subsequently monomers. The continuous loss of monomers will eventually lead to the phenomenon of erosion, which progressively changes the microstructure of the membrane through the formation of pores [31]. The combination of these processes could favor the possible application of our M4 and CNp-M4 membranes as wound dressings, because it would not be necessary to remove them from the application site, avoiding harm through injury and discomfort to the patient.

Moreover, the level of exudate from a wound (for example, an ulcer) can vary from absent (dry ulcer) to minimally exuding ( $<5 \mathrm{~mL}$ fluid per $24 \mathrm{~h}$ ), to moderately exuding ( 5 to $10 \mathrm{~mL}$ fluid per $24 \mathrm{~h}$ ), and finally to highly exuding ( $>10 \mathrm{~mL}$ fluid per $24 \mathrm{~h}$ ) [32]. In this regard, the measurement of the swelling capacity of a wound dressing developed with alginate could be classified as dressings of low absorbance (alginate wound dressing that absorbs less than $6 \mathrm{~g}$ of liquid per $\mathrm{g}$ of dressing, or less than $12 \mathrm{~g} / 100 \mathrm{~cm}^{2}$ ), and dressings of high absorbency (an alginate wound dressing that absorbs $6 \mathrm{~g}$ or more liquid per $\mathrm{g}$ of dressing, or $12 \mathrm{~g}$ or more $/ 100 \mathrm{~cm}^{2}$ ) [33]. With this consideration, CNp-M4 possesses a value of $17.48 \mathrm{~g} / 100 \mathrm{~cm}^{2}$, corresponding to high absorbency and similar to several commercial products.

\subsubsection{Mechanical Test}

Wound dressings must be resistant and flexible for ease of handling [17]. Thus, the mechanical properties of the membranes are depicted in Figure 3. Formulations with PVA (M1 and M2) did not demonstrate a significant difference in $\% \mathrm{E}$ with $73.54 \pm 0.87 \%$ and $74.90 \pm 1.67 \%$, respectively. TS was similar for M1, M2, and M3 samples with $1.32 \pm 0.02,1.34 \pm 0.01$, and $1.96 \pm 0.05 \mathrm{MPa}$, respectively. By way of comparison, M1 and M2 membranes exhibited a lower TS and \%E than the remaining formulations, probably due to PVA being a polymer that has been characterized as possessing poor elasticity, a rigid membrane, and low hydrophilic characteristics [34]. 
a)

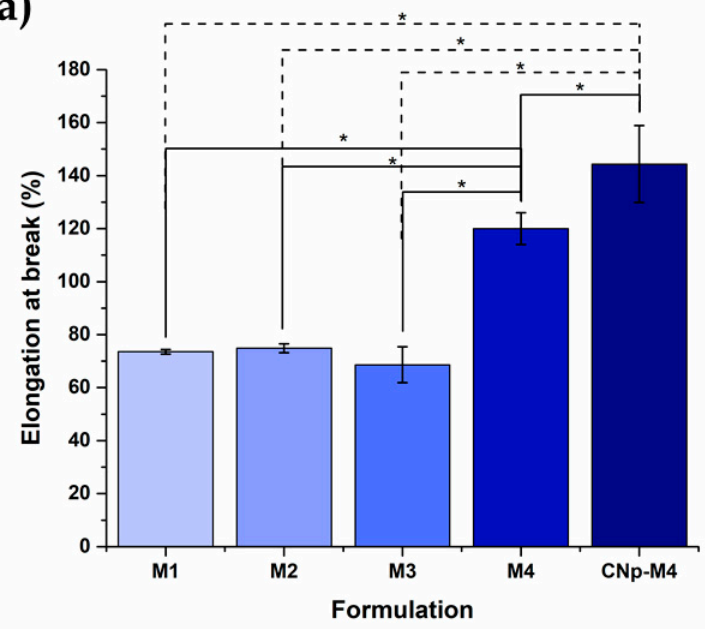

b)

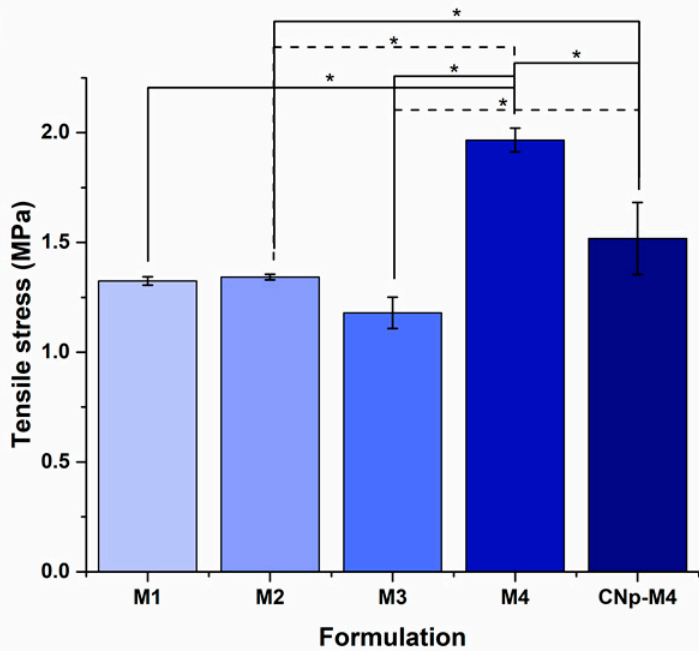

Figure 3. Effect of plasticizers on the mechanical properties of alginate membranes. Effect on mechanical properties by the addition of PVA + glycerin (M1), PVA + glycerin + propylene glycol (M2), PVP + glycerin (M3), PVP + glycerin + propylene glycol (M4), and CNp + PVP + glycerin + propylene glycol (CNp-M4), respectively. (a) Elongation at break; (b) tensile stress, (mean $\pm \mathrm{SD} ; n=3)$. ${ }^{*}$ indicates $p<$ 0.05 as statistically significant.

The $\% \mathrm{E}$ and TS of $\mathrm{M} 3$ were $68.63 \pm 6.75 \%$ and $1.18 \pm 0.07 \mathrm{MPa}$, respectively; these values significantly increased with the addition of propylene glycol (M4), to $120.01 \pm 5.97 \%$ and $1.96 \pm 0.05 \mathrm{MPa}$, respectively. M4 exhibited the highest values in the assay and showed a significant difference with respect to the remaining formulations without $\mathrm{CNp}$. This may be explained, at least in part, by the properties of propylene glycol, which is a plasticizer with a small molecular weight that is able to create multiple $\mathrm{H}$-bonds with PVP and SA chains into a package and, consequently, aid in the formation of cross-linked networks [35].

Therefore, because of its greater swelling capacity and better mechanical properties, the M4 membrane (a mixture of sodium alginate, PVP, and propylene glycol) was chosen for the incorporation of $\mathrm{CNp}$.

On the other hand, the mechanical properties of M4 were modified when $\mathrm{CNp}$ dispersion was added to the formulation. The \%E of CNp-M4 showed the highest value, with $144.39 \pm 14.52 \%$; in contrast, TS decreased to $1.52 \pm 0.16 \mathrm{MPa}$. This could be due to the addition of $\mathrm{CNp}$ dispersion to the formulation decreasing the number of hydrogen bonds between the polymer molecular chains; as a result, less strength is necessary to break the membrane [36]. In comparison, $\mathrm{CNp}-\mathrm{M} 4$ showed the highest $\% \mathrm{E}$, which could be due to the effect of Pluronic ${ }^{\circledR} \mathrm{F}-68$. The latter is a surfactant that decreases the pore number, providing a membrane with a homogeneous structure; thus, it is more resistant to changes, rendering higher elasticity properties.

\subsubsection{Thermogravimetric Analysis (TGA)}

It is important to determine the thermal properties of a substance, because these provide useful information for their identification and the characterization of materials. In Figure 4, thermograms of curcumin, $\mathrm{CNp}, \mathrm{M} 4$, and $\mathrm{CNp}-\mathrm{M} 4$ membranes are presented. For curcumin, mass loss was observed at $173^{\circ} \mathrm{C}$ by TGA (Figure 4a); due to the degradation of turmeric powder, water loss was not observed, possibly due to its high hydrophobicity [37]. The weight loss of CNp started at approximately $90^{\circ} \mathrm{C}$, corresponding to dehydration, and there was a second plateau from 280 to $350^{\circ} \mathrm{C}$, suggesting a better thermal stability for curcumin when it is inside PCL nanoparticles than when alone. However, CNp lost more weight in a smaller temperature range. 

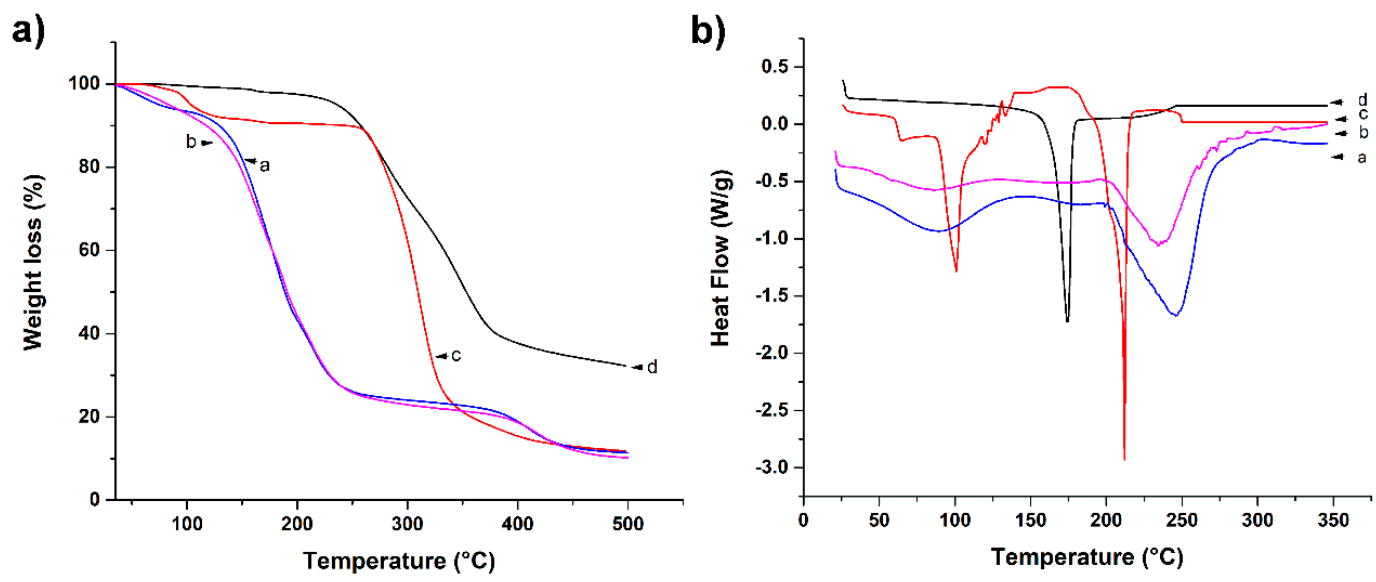

Figure 4. Thermal analysis of nanoparticle-coated alginate membrane using (a) thermogravimetric analysis (TGA) and (b) differential scanning calorimetry (DSC). The thermal properties of the M4 membrane, CNp-M4 membrane, $\mathrm{CNp}$, and curcumin are represented as a, b, c, and d, respectively.

On the other hand, in the M4 membrane, the first mass loss occurred between 90 and $240{ }^{\circ} \mathrm{C}$, whereas for the $\mathrm{CNp}-\mathrm{M} 4$ sample, weight loss began between 100 and $240{ }^{\circ} \mathrm{C}$. This could be due to the evaporation of water traces, the degradation of propylene glycol, PVP, glycerin $\left(150-220^{\circ} \mathrm{C}\right)$, and turmeric powder [38]. In the case of the $\mathrm{CNp}-\mathrm{M} 4$ membrane, it presented a slighter weight loss compared to the M4 membrane. The second mass loss of both samples was between 270 and $425^{\circ} \mathrm{C}$; in this stage, the decomposition of the functional groups of SA polymer chains is presented. Finally, the last plateau in M4 and CNp-M4 membranes started at $425^{\circ} \mathrm{C}$, which corresponds to the degradation of the SA backbone [19]. In the same manner, the CNp-M4 membrane thermogram revealed a lower weight-loss temperature compared with that of $\mathrm{CNp}$; this is probably because membrane formulation is a mixture that contains more substances than $\mathrm{CNp}$. This is similar to the thermal behavior exhibited by curcumin and CNp.

\subsubsection{Differential Scanning Calorimetry (DSC)}

DSC is a technique used to determine the quantity of heat either absorbed or released when substances undergo physical or chemical changes [39]. In Figure 4b, DSC thermograms of curcumin, $\mathrm{CNp}$, M4 membrane as vehicle, and CNp-M4 membrane are presented. The melting point of curcumin was found to be $174{ }^{\circ} \mathrm{C}$ (Table 2), which was expected with regard to the literature [40]. Furthermore, three thermal events were observed in the $\mathrm{CNp}$ : at $63.5 ; 101$, and $212{ }^{\circ} \mathrm{C}$. The first thermal event could correspond to the melting point of PCL $\left(61^{\circ} \mathrm{C}\right)$ [41], while events at 101 and $212{ }^{\circ} \mathrm{C}$ may indicate the presence of trehalose, which was employed as a cryoprotectant to lyophilize the $\mathrm{CNp}$ [42]. Interestingly, the melting point of curcumin was not detected in the CNp sample; this could be due to the high $\mathrm{EE}$ of curcumin inside PCL nanoparticles as a molecular dispersion.

Table 2. Thermal events of curcumin, CNp, and alginate membranes by DSC.

\begin{tabular}{cccc}
\hline Sample & $\mathrm{T}_{\mathbf{m} \mathbf{1}}\left({ }^{\circ} \mathrm{C}\right)$ & $\mathrm{T}_{\mathbf{m} \mathbf{2}}\left({ }^{\circ} \mathrm{C}\right)$ & $\mathrm{T}_{\mathbf{m} 3}\left({ }^{\circ} \mathrm{C}\right)$ \\
\hline Curcumin & 174 & - & - \\
CNp & 63.5 & 101 & 212 \\
M4 & 87 & 249 & - \\
CNp-M4 & 87 & 233 & - \\
\hline
\end{tabular}

On the other hand, both M4 and CNp-M4 membranes revealed two thermal events. The first peak was around $87^{\circ} \mathrm{C}$ for both formulations, whereas the second peak was around $233^{\circ} \mathrm{C}$ for the M4 membrane and $249^{\circ} \mathrm{C}$ for the $\mathrm{CNp}-\mathrm{M} 4$ membrane. The latter peaks were due to the presence of SA in 
the formulation. A thermal peak prior to $100{ }^{\circ} \mathrm{C}$ was observed for both samples, possibly due to the presence of water in the membranes.

\subsection{5. $\mathrm{pH}$ Determination}

The $\mathrm{pH}$ values of all the polymer gels were $5.78 \pm 0.06,5.76 \pm 0.03,5.65 \pm 0.01,5.97 \pm 0.05$ and $5.68 \pm 0.03$ for M1, M2, M3, M4, and CNp-M4, respectively. All of these values are acceptable because $\mathrm{pH}$ wound dressings must be neither acid nor alkaline in order to avoid skin irritation. Moreover, membrane $\mathrm{pH}$ is important to regulate the wound-healing process. The natural $\mathrm{pH}$ of the skin is within a range of 5-6, depending on the person, while the $\mathrm{pH}$ of the chronic wound oscillates in an alkaline range between $\mathrm{pH} 7$ and 8 , which increases susceptibility to wound infection.

\subsubsection{Structure and Morphology of M4 and CNp-M4 Membranes}

An ideal scaffold is expected to have a suitable microstructure (number of pores and pore size controlled) in order to transport nutrients, cells, metabolites, gases, and signaling molecules [43]. In this respect, pores were observed in the top of the membrane structure, which did not span the membrane (Figure 5a). However, it should be expected that the addition of water to the membranes (for example, from the wound exudate) promotes the total formation of pores through these. This would allow skin transpiration and an optimal environment for the wound.

a)

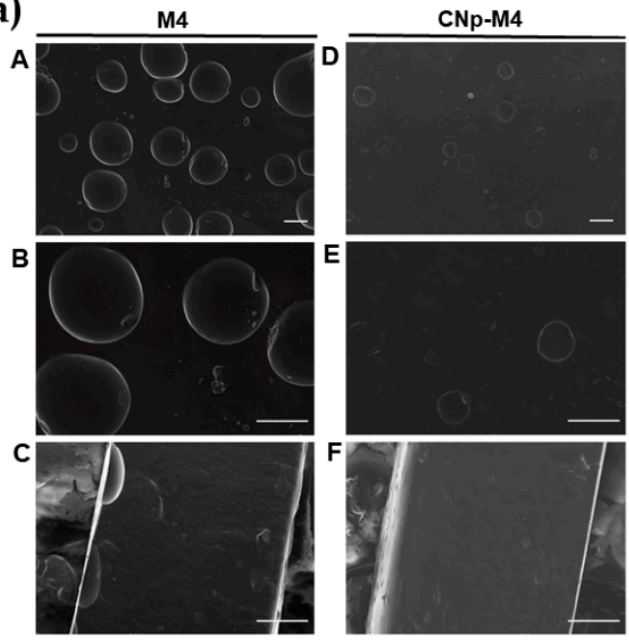

b)

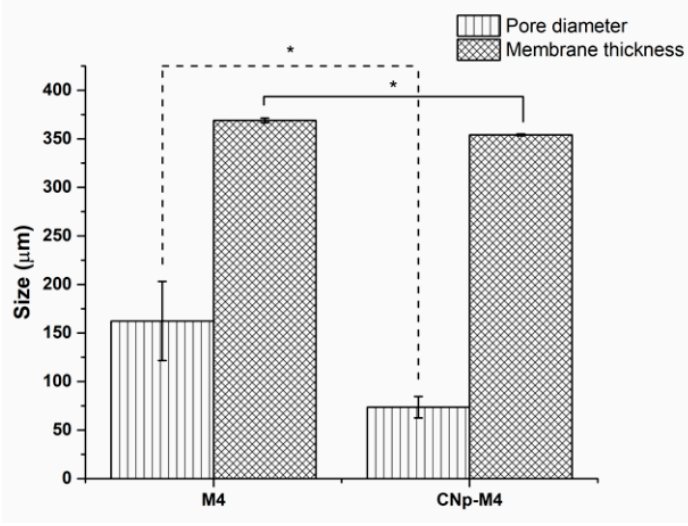

Figure 5. Morphology and porosity of alginate membranes. (a) Micrographs by scanning electronic microscopy of the alginate membrane surface (A, B, D, and E) and membrane thickness (C and F). Magnification of 100× for A, D; 220× in B, C, E and F; the scale bar is $100 \mu \mathrm{m}$; (b) pore diameter and membrane thickness of M4 and CNp-M4 membranes, mean $\pm \mathrm{SE}, n=3 .{ }^{*}$ indicates that $p<0.05$ is statistically significant.

On the other hand, the mean pore numbers in M4 and CNp-M4 membranes found in each $0.199 \mathrm{~mm}^{2}$ were 4 and 2, respectively. The pore diameter in the M4 membrane was $162.25 \pm 40.75 \mu \mathrm{m}$, while for $\mathrm{CNp}-\mathrm{M} 4$ membranes, this was $73.43 \pm 11.04 \mu \mathrm{m}$ (Figure $5 \mathrm{~b}$ ). Moreover, M4 membranes were significantly thicker and more homogenous in structure than CNp-M4 membranes. These features could be due to the fact that $\mathrm{CNp}-\mathrm{M} 4$ membranes have $\mathrm{CNp}$ dispersion in their formulation, which contains Pluronic ${ }^{\circledR}$ F-68, a nonionic surfactant used as a nanoparticle coating that decreases tensile surface in the polymer gel, thus reducing the number and size of the pores formed in the membranes [43].

Finally, transparency is an expected feature in our alginate membranes used as wound dressings, in order to observe the possible wound-healing process without removing the dressing. M4 and $\mathrm{CNp}-\mathrm{M} 4$ membranes revealed a transparent feature prior to swelling, while $\mathrm{CNp}-\mathrm{M} 4$ membranes 
demonstrated a translucent feature during the swelling process (Figure 6). Although the latter was not completely transparent, it was possible to see through it.
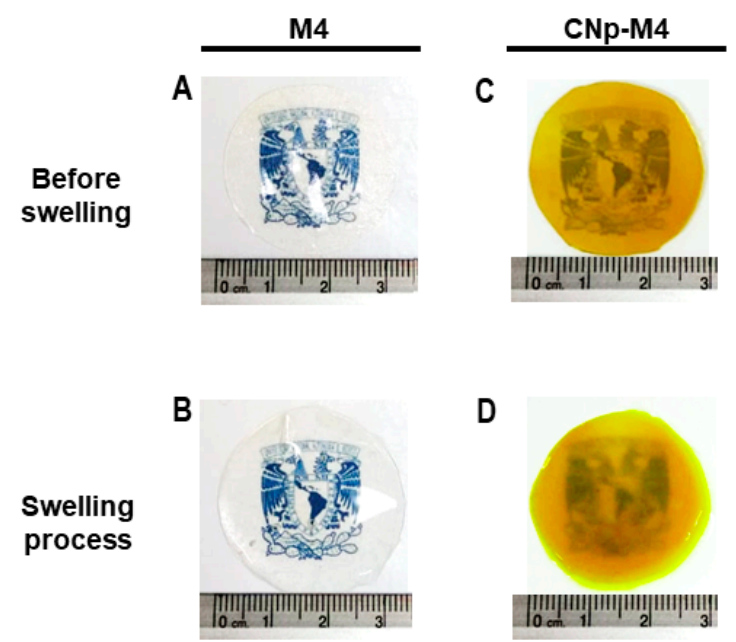

Figure 6. Alginate membranes (M4) before swelling and during the swelling process in PBS medium (A,B, respectively), and alginate membranes with curcumin nanoparticles (CNp-M4) before swelling and during the swelling process in PBS medium (C,D, respectively). Scale in centimeters.

\subsubsection{In Vitro Release Study of Drug Dispersion, $\mathrm{CNp}$ and CNp-M4}

To analyze the mechanism of drug release from the nanoparticles $(\mathrm{CNp})$ and from the nanoparticles inside the membrane (CNp-M4), an in vitro release study was performed via the dispersion method and is presented in Figure 7. The curcumin release from CNp (red line, circle symbol) showed a low burst effect at $2 \mathrm{~h}$; this behavior could be related to the presence of the curcumin released from the nanoparticles, as well as to the curcumin outside the nanoparticle, inside the border-zone matrix-stabilizer. After that, the release profile exhibited a linear behavior, with nearly $60 \%$ of the curcumin released after $48 \mathrm{~h}$ of study. Interestingly, in the release profile for $\mathrm{CNp}-\mathrm{M} 4$ (blue line, triangle symbol), the burst effect was not evident, and a faster release than $\mathrm{CNp}$ ( $80 \%$ of curcumin released at $48 \mathrm{~h}$ ) was found. These behaviors may be due to the interaction among the membrane excipients, the solvents, and the nanoparticles. To elaborate the CNp-M4 membrane (Section 2.4.1), the nanoparticles are in contact with water, and the hydrolysis of $\mathrm{CNp}$ could be stimulated. In addition, some excipients, such as glycerin and propylene glycol, are co-solvents that could improve the prior solubilization of curcumin. As can be observed, the release from both the $\mathrm{CNp}$ and $\mathrm{CNp}-\mathrm{M} 4$ membrane was considerably slower than the drug dispersion release (black line, square symbol).

In order to investigate the mechanism of curcumin release from $\mathrm{CNp}$ and $\mathrm{CNp}-\mathrm{M} 4$, different mathematical models were applied (Table 3). The $\mathrm{CNp}$ data were fixed with the Higuchi model $\left(\mathrm{A}=0.0852, \mathrm{~B}=-0.0459, R^{2}=0.9551\right)$, according to those previously reported [44]. This model describes the release of the drug by diffusion from the nanoparticle core into the external solution. On the other hand, the release from $\mathrm{CNp}-\mathrm{M} 4$ could be explained with the Korsmeyer-Peppas model, due to the highest squared-correlation-coefficient value being obtained with this method $(\mathrm{A}=0.3119, \mathrm{~B}=-0.5609$, $\left.R^{2}=0.9536\right)$. This model combines the diffusion and erosion mechanisms of the nanoparticles as the explanation for drug release. 


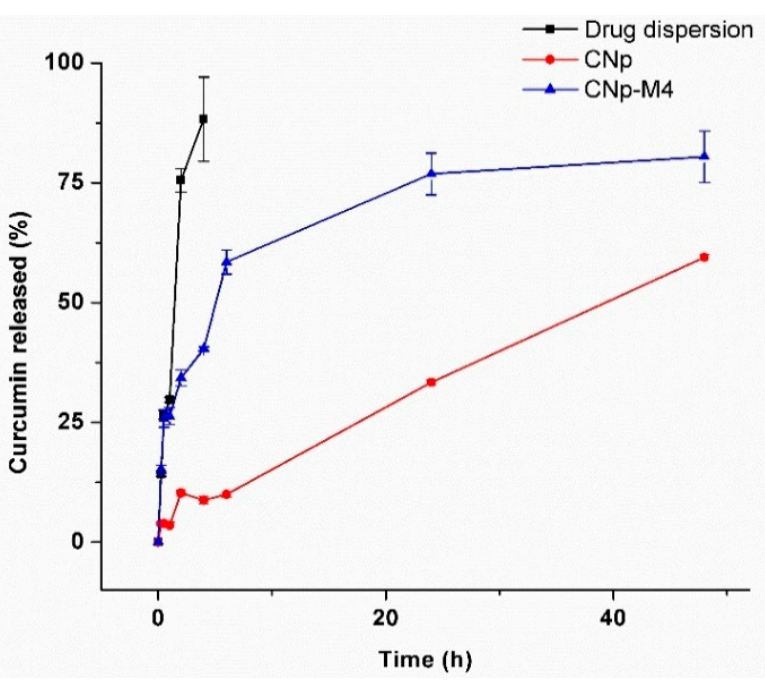

Figure 7. Release profile of curcumin from the drug dispersion, $\mathrm{CNp}$ and $\mathrm{CNp}-\mathrm{M} 4$ membrane in PBS pH 7.4 (0.1 M, Pluronic $\left.{ }^{\circledR} \mathrm{F}-1272 \% w / v\right)$ at $37^{\circ} \mathrm{C}$. Each point represents the mean $\pm \mathrm{SE}, n=3$.

Table 3. Squared of the correlation coefficient $\left(R^{2}\right)$ and coefficients obtained after the linear regression of release data from $\mathrm{CNp}$ and CNP-M4 utilizing four mathematical models.

\begin{tabular}{cccccccc}
\hline \multirow{2}{*}{$\begin{array}{c}\text { Mathematical } \\
\text { Model }\end{array}$} & Equation & \multicolumn{3}{c}{ CNp } & \multicolumn{3}{c}{ CNp-M4 } \\
\cline { 3 - 7 } & & $\boldsymbol{R}^{\mathbf{2}}$ & $\mathbf{A}$ & $\mathbf{B}$ & $\boldsymbol{R}^{\mathbf{2}}$ & $\mathbf{A}$ & $\mathbf{B}$ \\
\hline Zero-order & $\mathrm{Q}_{\mathrm{t}}=\mathrm{Q}_{0}+\mathrm{K}_{0} \mathrm{t}$ & 0.842 & 0.5264 & 4.7385 & 0.7358 & 1.2362 & 31.432 \\
\hline First-order & $\ln _{\mathrm{t}}=\mathrm{loQ}_{0}+\mathrm{K}_{1} \mathrm{t}$ & 0.8436 & -0.0066 & -2.3991 & 0.8453 & -0.0303 & -3.1975 \\
\hline Higuchi & $\mathrm{Q}_{\mathrm{t}}=\mathrm{K}_{\mathrm{H}} \mathrm{t}^{1 / 2}$ & 0.9551 & 0.0852 & -0.0459 & 0.8939 & 0.1017 & 0.194 \\
\hline Korsmeyer-Peppas & $\mathrm{Q}_{\mathrm{t}}=\mathrm{K}_{\mathrm{K}} \mathrm{t}^{\mathrm{n}}$ & 0.9037 & 0.5422 & -1.2655 & 0.9536 & 0.3119 & -0.5609 \\
\hline
\end{tabular}

$\mathrm{Q}_{\mathrm{t}}$ : amount of drug released in time $\mathrm{t} ; \mathrm{Q}_{0}$ : initial amount of drug in the dosage form; $\mathrm{Q}_{\infty}$ : total amount of drug dissolved when the dosage form is exhausted; $\mathrm{K}_{0}, \mathrm{~K}_{1}, \mathrm{~K}_{\mathrm{H}}, \mathrm{K}_{\mathrm{K}}$ : release rate constants; $R^{2}$ : squared correlation coefficient. $y=a x \pm b$ is an equation obtained after regression: $a$, slope and $b$, linear coefficient.

\subsection{Curcumin Permeation Assays}

\subsubsection{Ex Vivo Permeation}

An ideal system for potential use in chronic diseases with a slow healing process, such as wounds or psoriasis, should exhibit sustained drug release in order to allow permeation through the skin [20,45]. In this regard, the alginate membranes developed in our study possess polymeric networks as a structure, as well as the curcumin encapsulated in PCL nanoparticles dispersed within these networks. These features will allow the slow release of curcumin.

An ex vivo permeation study was conducted to determine the distribution of the drug and $\mathrm{CNp}$ throughout the stratum corneum, epidermis, and dermis, and to verify whether curcumin can pass through the skin and reach blood circulation after the administration of $\mathrm{CNp}-\mathrm{M} 4$ and $\mathrm{CNp}$ formulations. With respect to the aqueous dispersion of curcumin (Figure 8), due to the high lipophilic character of the drug, a high accumulation was observed in some superficial layers of the stratum corneum $\left(10.04 \pm 1.73 \mu \mathrm{g} / \mathrm{cm}^{2}\right)$. Interestingly, permeation comprises a considerable amount, even from the application of a curcumin dispersion in water, which involves solid particle clusters. This means that the drug particles engage in a dissolution process with the oily components of the stratum corneum, leading to their brief permeation in the superficial region. Although the values are high, there is a higher efficiency of permeation with $\mathrm{CNp}\left(14.80 \pm 1.61 \mu \mathrm{g} / \mathrm{cm}^{2}\right)$. In the region of the dermis, with a hydrophilic character, the permeated amount of the drug dispersion decreases considerably due to its 
highly limited solubility in aqueous media $\left(2.40 \pm 0.46 \mu \mathrm{g} / \mathrm{cm}^{2}\right)$; this is nearly one third in relation to the $\mathrm{CNp}$ value $\left(6.99 \pm 0.27 \mu \mathrm{g} / \mathrm{cm}^{2}\right)$. No detection was recorded for the dispersion of the drug that could completely permeate the skin. According to Figure 8, a significant difference was observed between $\mathrm{CNp}$ dispersion and CNp-M4 membrane treatments, with the highest permeation values observed for $\mathrm{CNp}$. The CNp-M4 membrane treatment revealed the highest amount of curcumin retained in the epidermis and dermis - that is, $5.7 \mu \mathrm{g} / \mathrm{cm}^{2}(1.620 \pm 0.051 \%$ of the total concentration of curcumin) - and the lowest concentration was found in the stratum corneum (SC), at $0.65 \mu \mathrm{g} / \mathrm{cm}^{2}(0.140 \pm 0.006 \%)$. On the other hand, in the CNp dispersion treatment, curcumin was found mostly in the SC; that is, $14.8 \mu \mathrm{g} / \mathrm{cm}^{2}(2.62 \pm 0.49 \%)$ [26], the highest curcumin value in the entire assay.

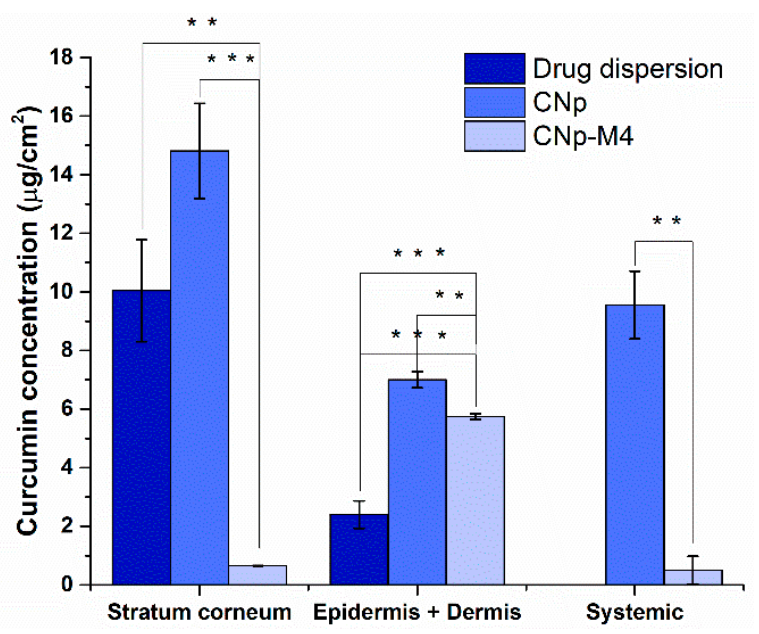

Figure 8. Ex vivo permeation of curcumin after $30 \mathrm{~h}$ of treatment with drug dispersion, CNp dispersion, or CNp-M4 membrane. Stratum corneum-bound particles (obtained from 15 tape strips), epidermis + dermis (surface on which dosed skin was handled after $30 \mathrm{~h}$ ), and systemic (receptor compartment), mean $\pm \mathrm{SE}, n=4{ }^{* *}$ indicates $p<0.01$ and ${ }^{* * *}$ indicates $p<0.001$ as statistically significant.

There was also a significant difference in the amount of curcumin that crossed through the skin (which, in an in vivo model, means reaching the systemic circulation), since the concentration derived from the CNp-M4 membrane was significantly lower than $\mathrm{CNp}(0.32 \%$ and $2.04 \%$ respectively; $p$-value $=0.0019$ ).

The aqueous system of $\mathrm{CNp}$ dispersion permitted the curcumin to permeate through the dermis and completely cross the skin. Moreover, CNps have a negative charge; negatively charged nanoparticles permeate the skin more rapidly than positively charged nanoparticles. The skin is predominately negatively charged, and the electrostatic interaction of positive particles with the negatively charged molecules in the skin matrix slows particle diffusion [26]. However, curcumin from CNp-M4 membranes diffuses more slowly through the skin than that from $\mathrm{CNp}$. These observations may be due to the fact that, in the CNp dispersion, water was used as the medium. Water affects the absorption rates of different substances through the stratum corneum, which is in a constant state of partial hydration under normal conditions. Thus, when immersed in water, dead keratinocytes quickly absorb it, resulting in the pruning effect of the skin [45]. Furthermore, water in contact with skin creates a flow gradient toward the skin's inner layers, since the inside of the stratum corneum is more hydrated than the surface [46]. On the other hand, the CNp-M4 membrane does not have a liquid medium in the interface that allows the nanoparticles to flow easily into the deep layers, such as water in the case of the CNp dispersion. Despite this, for the CNp-M4 membrane, a modulated release is expected because of the degree of swelling of the membrane in response to the presence of exudate. The swelling would permit the relaxation of the polymer chains and the release from the $\mathrm{CNp}$. Otherwise, diffusion from a solid state (CNp-M4) into a semi-solid state (the skin) would be expected. Therefore, the CNp-M4 membrane represents a prolonged release system. 
In addition, the skin possesses furrows, in which a considerable amount of curcumin is retained, which could not be extracted with the application of adhesive tapes [47]. This curcumin was quantified until mechanical disaggregation. This could explain why the epidermis and dermis had the highest concentration of the drug.

On the other hand, particle size is an important factor in obtaining the desired therapeutic effect, because nanoparticles with a small size can more easily permeate the physiological barriers; moreover, due to their greater surface, release of the drug is favored [48]. Thus, it should be expected that CNps, with their small size $(148.3 \mathrm{~nm})$, and the surfactant effect of Pluronic ${ }^{\circledR}$ F-68 can permeate intercellularly and through hair follicles, favoring accumulation for several hours. In the same way, due to the contact of nanoparticles with the corneocytes of the skin, as well as the prolonged release thereof, a large amount of curcumin was found to be retained in the dermis [24,27]. Although after $30 \mathrm{~h}$ the majority of curcumin remained in the stratum corneum when $\mathrm{CNp}$ dispersion was applied, the monitoring of the permeation at longer times could allow the observation of a prolonged release system [49]. Therefore, the $\mathrm{CNp}-\mathrm{M} 4$ membrane is proposed as a functional prolonged release system for drug delivery in chronic diseases; however, it would be necessary to perform a more prolonged test to observe the diffusion of the drug at a greater proportion.

\subsubsection{Permeation Assay in Vivo}

In order to evaluate the in vivo skin permeation of curcumin from the $\mathrm{CNp}-\mathrm{M} 4$ membrane, $\mathrm{CNp}$ dispersion [20], and drug dispersion, a quantification of the curcumin deposited in the stratum corneum by UV-Vis spectrophotometry was performed. The results are presented in Figure 9, according to the work of Goto et al. [50].

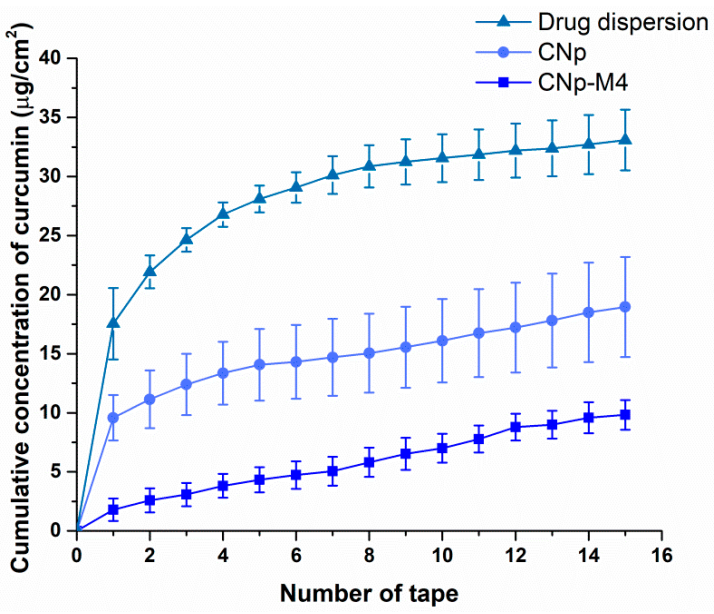

Figure 9. Cumulative concentration of curcumin quantified in the stratum corneum of healthy volunteers. Drug extraction from 15 adhesive tapes applied to the treatment site after placing a CNp-M4 membrane, $\mathrm{CNp}$ dispersion, or drug dispersion for $6 \mathrm{~h}($ mean $\pm \mathrm{SE} ; n=4)$.

Higher permeation values were observed for the drug dispersion in water compared to the $\mathrm{CNp}$ and CNp-M4 membrane, at least in the superficial layers of the stratum corneum, where the tape stripping technique is applied. The higher values of drug permeation could correspond to the high lipophilicity value of the drug, structural symmetry, and low molecular weight. These values coincide with Figure 8: greater drug deposition on the stratum corneum surface, a lower proportion in the dermis due to an inadequate hydrophilic-lipophilic balance, and no recorded quantity that completely permeates the skin. At $6 \mathrm{~h}$ after the application of the drug dispersion, CNp-M4 membrane, and $\mathrm{CNp}$ dispersion, the curcumin measured in the stratum corneum reached $33.08 \pm 2.57,9.82 \pm 4.23$, and $18.96 \pm 1.25 \mu \mathrm{g} / \mathrm{cm}^{2}$, respectively [25]. CNp-M4 remained well adhered during the study, even up to $48 \mathrm{~h}$ in other volunteers (data not shown). As can be noted, a greater amount of curcumin was observed in the stratum corneum when the $\mathrm{CNp}$ dispersion treatment was applied, compared with the 
$\mathrm{CNp}-\mathrm{M} 4$ membrane. This is because, in the $\mathrm{CNp}-\mathrm{M} 4$ membrane, the nanoparticles must be released from the polymeric matrix. This result suggests our formulation as a system of prolonged release that may be useful for long treatments, such as that for a wound (for example, for 7-14 days of application until closure of the lesion). It is noteworthy that the smallest variation observed in permeation values with the $\mathrm{CNp}-\mathrm{M} 4$ treatment reveals a system that allows better gradual control release. Moreover, these nanoparticles also possess a stabilizer on the outside, Pluronic ${ }^{\circledR}$ F-68, which interacts through hydrogen bonds with the -OH groups of plasticizers and polymers used in the formulation. Thus, when the water makes contact with the membrane, the external part of the polymers swells and promotes the drug — in this case, $\mathrm{CNp}$ - to flow outward, permitting its release [51].

With respect to the $\mathrm{CNp}$ dispersion, the nanoparticles are free in the medium; thus, they interact more easily with the stratum corneum. Curcumin is encapsulated and uniformly distributed in the PCL nanoparticles, forming nanospheres [52]. The release of curcumin from these latter will depend on the solubility of the drug, the diffusion of curcumin through the matrix of the nanoparticles, thedesorption of curcumin from PCL, the erosion or degradation of the matrix of the nanoparticles, and on the combination of the erosion and diffusion processes [23]. It is also known that, under physiological conditions, a random cleavage of PCL ester bonds occurs, which produces a destabilization of the polymer matrix of the $\mathrm{CNp}$, inducing the release of curcumin [25].

Finally, many studies have reported an accumulation of nanoparticles in hair follicles and the pilosebaceous glands in ex vivo skin experiments [49]. In the same manner, it has been reported that there is better permeation of the drug into the skin, as well as greater absorption in areas with high hair-follicle density. The hair follicle can become a reservoir of substances comparable to the stratum corneum [53]. This means that drugs will penetrate better through the skin of a person with greater amounts of hair follicles. This may explain slight variations in the results obtained, together with variability among individuals (age, body mass, color, and skin moisturization).

\section{Conclusions}

In this study, the development of a new wound dressing for possible application in wound healing was demonstrated. The wound dressing comprises an alginate membrane and PCL nanoparticles stabilized with Pluronic ${ }^{\circledR}$ F-68 with curcumin inside. This new system was designed with a mixture of aqueous plasticizers to confer high-strength mechanical properties. The characterization of the formulation exhibited that it possesses a high absorbency capacity for the removal of possible exudates, transparency for monitoring the wound bed, the presence of pores with controlled dimensions that could facilitate the transpiration of the wound as a synthetic skin substitute, and the gradual release of the drug according to the ex vivo and in vivo studies. In addition, the high adherence of the wound dressing should be noted even in dry skin, as well as its degradation at later times. Therefore, all of these advantages reveal our wound dressing as a good option for wound healing without the need for its removal from the patient.

Author Contributions: Conceptualization, G.L.-G.; methodology, M.C.G.-A., R.A.M.-F., and M.L.D.P.-A.; validation, I.H.C.-F., C.D.F.-H., and B.F.; formal analysis, M.C.G.-A., R.A.M.-F., M.L.D.P.-A., and G.L.-G.; investigation, M.C.G.-A., R.A.M.-F., and G.L.-G.; resources, M.C.G.-A., R.A.M.-F., M.L.D.P.-A., and G.L.-G.; data curation, M.C.G.-A., R.A.M.-F., M.L.D.P.-A., Z.U.-M., H.C., and G.L.-G.; writing-original draft preparation, M.C.G.-A., R.A.M.-F., M.L.D.P.-A., Z.U.-M., H.C., and G.L.-G.; writing-review and editing, M.C.G.-A., R.A.M.-F., Z.U.-M., D.M.G.-G., J.J.M., M.G.-T., O.D.R.-H., G.F.-G., I.H.C.-F., C.D.F.-H., B.F., H.C., and G.L.-G.; visualization, G.L.-G.; supervision, M.L.D.P.-A., Z.U.-M., H.C., and G.L.-G.; project administration, M.L.D.P.-A. and G.L.-G.; funding acquisition, G.L.-G.

Funding: This research was funded by Dirección General de Asuntos del Personal Académico, Universidad Nacional Autónoma de México (Becas Posdoctorales, PAPIIT TA 200318) and CONACYT A1-S-15759.

Conflicts of Interest: The authors declare no conflict of interest. 


\section{References}

1. Eming, S.A.; Brachvogel, B.; Odorisio, T.; Koch, M. Regulation of angiogenesis: Wound healing as a model. Prog. Histochem. Cytochem. 2007, 42, 115-170. [CrossRef] [PubMed]

2. Demidova-Rice, T.N.; Hamblin, M.R.; Herman, I.M. Current Methods for Drug Delivery, Part 1: Normal and Chronic Wounds: Biology, Causes, and Approaches to Care. Adv. Skin Wound Care 2013, 25, 304-314. [CrossRef] [PubMed]

3. Axibal, E.; Brown, M. Surgical Dressings and Novel Skin Substitutes. Dermatol. Clin. 2019, 37, $349-366$. [CrossRef] [PubMed]

4. Aderibigbe, B.A.; Buyana, B. Alginate in wound dressings. Pharmaceutics 2018, 10, 42. [CrossRef] [PubMed]

5. Morgado, P.I.; Aguiar-Ricardo, A.; Correia, I.J. Asymmetric membranes as ideal wound dressings: An overview on production methods, structure, properties and performance relationship. J. Memb. Sci. 2015, 490, 139-151. [CrossRef]

6. Mi, F.L.; Wu, Y.B.; Shyu, S.S.; Chao, A.C.; Lai, J.Y.; Su, C.C. Asymmetric chitosan membranes prepared by dry/wet phase separation: A new type of wound dressing for controlled antibacterial release. J. Memb. Sci. 2003, 212, 237-254. [CrossRef]

7. Priya, S.G.; Gupta, A.; Jain, E.; Sarkar, J.; Damania, A.; Jagdale, P.R.; Chaudhari, B.P.; Gupta, K.C.; Kumar, A. Bilayer Cryogel Wound Dressing and Skin Regeneration Grafts for the Treatment of Acute Skin Wounds. ACS Appl. Mater. Interfaces 2016, 8, 15145-15159. [CrossRef]

8. Miguel, S.P.; Moreira, A.F.; Correia, I.J. Chitosan based-asymmetric membranes for wound healing: A review. Int. J. Biol. Macromol. 2019, 127, 460-475. [CrossRef]

9. Sood, A.; Granick, M.S.; Tomaselli, N.L. Wound Dressings and Comparative Effectiveness Data. Adv. Wound Care 2014, 3, 511-529. [CrossRef]

10. Aggarwal, B.B.; Harikumar, K.B. Potential therapeutic effects of curcumin, the anti-inflammatory agent, against neurodegenerative, cardiovascular, pulmonary, metabolic, autoimmune and neoplastic diseases. Int. J. Biochem. Cell Biol. 2009, 41, 40-59. [CrossRef]

11. Augustyniak, A.; Bartosz, G.; Čipak, A.; Duburs, G.; Horáková, L.; Łuczaj, W.; Majekova, M.; Odysseos, A.D.; Rackova, L.; Skrzydlewska, E.; et al. Natural and synthetic antioxidants: An updated overview. Free Radic. Res. 2010, 44, 1216-1262. [CrossRef]

12. Del Prado-Audelo, M.L.; Caballero-Florán, I.H.; Meza-Toledo, J.A.; Mendoza-Muñoz, N.; González-Torres, M.; Florán, B.; Cortés, H.; Leyva-Gómez, G. Formulations of curcumin nanoparticles for brain diseases. Biomolecules 2019, 9, 56. [CrossRef]

13. Sidhu, G.S.; Singh, A.K.; Thaloor, D.; Banaudha, K.K.; Patnaik, G.K.; Srimal, R.C.; Maheshwari, R.K. Enhancement of wound healing by curcumin in animals. Wound Repair Regen. 1998, 6, 167-177. [CrossRef]

14. Thangapazham, R.L.; Sharad, S.; Maheshwari, R.K. Skin regenerative potentials of curcumin. Biofactors 2013, 39, 141-149. [CrossRef]

15. Yallapu, M.M.; Jaggi, M.; Chauhan, S.C. Curcumin nanoformulations: A future nanomedicine for cancer. Drug Discov. Today 2012, 17, 71-80. [CrossRef]

16. Quintanar-guerrero, D.; Allémann, E.; Fessi, H.; Doelker, E.; Allémann, E.; Fessi, H.; Doelker, E. Preparation Techniques and Mechanisms of Formation of Biodegradable Nanoparticles from Preformed Polymers. Drug Dev. Ind. Pharm. 1998, 24, 1113-1128. [CrossRef]

17. Karki, S.; Kim, H.; Na, S.; Shin, D.; Jo, K.; Lee, J. Thin films as an emerging platform for drug delivery. Asian J. Pharm. Sci. 2016, 11, 559-574. [CrossRef]

18. Golafshan, N.; Rezahasani, R.; Tarkesh Esfahani, M.; Kharaziha, M.; Khorasani, S.N. Nanohybrid hydrogels of laponite: PVA-Alginate as a potential wound healing material. Carbohydr. Polym. 2017, 176, 392-401. [CrossRef]

19. Song, Y.; Jiang, Z.; Gao, B.; Wang, H.; Wang, M.; He, Z.; Cao, X.; Pan, F. Embedding hydrophobic MoS2nanosheets within hydrophilic sodium alginate membrane for enhanced ethanol dehydration. Chem. Eng. Sci. 2018, 185, 231-242. [CrossRef]

20. Mao, K.; Fan, Z.; Yuan, J.; Chen, P.; Yang, J.; Xu, J. Skin-penetrating polymeric nanoparticles incorporated in silk fibroin hydrogel for topical delivery of curcumin to improve its therapeutic effect on psoriasis mouse model. Colloids Surf. B Biointerfaces 2017, 160, 704-714. [CrossRef] 
21. Kamar, S.S.; Abdel-Kader, D.H.; Rashed, L.A. Beneficial effect of Curcumin Nanoparticles-Hydrogel on excisional skin wound healing in type-I diabetic rat: Histological and immunohistochemical studies. Ann. Anat. 2019, 222, 94-102. [CrossRef]

22. Clayton, K.N.; Salameh, J.W.; Wereley, S.T.; Kinzer-Ursem, T.L. Physical characterization of nanoparticle size and surface modification using particle scattering diffusometry. Biomicrofluidics 2016, 10, 1-14. [CrossRef]

23. Singh, R.; Lillard, J.W., Jr. Nanoparticle-based targeted drug delivery. Exp. Mol. Pathol. 2009, 86, $215-223$. [CrossRef]

24. Moghimi, S.M.; Hunter, A.C. Good Review on Poloxamers and Poloxamines in Pharma. Elsevier 2000, 18, 412-420.

25. Ramanujam, R.; Sundaram, B.; Janarthanan, G.; Devendran, E.; Venkadasalam, M.; John Milton, M.C. Biodegradable Polycaprolactone Nanoparticles Based Drug Delivery Systems: A Short Review. Biosci. Biotechnol. Res. Asia 2018, 15, 679-685. [CrossRef]

26. Kraeling, M.E.K.; Topping, V.D.; Keltner, Z.M.; Belgrave, K.R.; Bailey, K.D.; Gao, X.; Yourick, J. In vitro percutaneous penetration of silver nanoparticles in pig and human skin. Regul. Toxicol. Pharmacol. 2018, 95, 314-322. [CrossRef]

27. Quintanar-Guerrero, D.; de la Luz Zambrano-Zaragoza, M.; Gutierrez-Cortez, E.; Mendoza-Munoz, N. Impact of the emulsification-diffusion method on the development of pharmaceutical nanoparticles. Recent Pat. Drug Deliv. Formul. 2012, 6, 184-194. [CrossRef]

28. Pinto Reis, C.; Neufeld, R.J.; Ribeiro, A.J.; Veiga, F.; Nanoencapsulation, I. Methods for preparation of drug-loaded polymeric nanoparticles. Nanomed. Nanotechnol. Biol. Med. 2006, 2, 8-21. [CrossRef]

29. Judefeind, A.; de Villiers, M.M. Nanotechnology in Drug Delivery; de Villiers, M.M., Aramwit, P.S., Kwon, G., Eds.; Springer US: New York, NY, USA, 2009; ISBN 9780387776675.

30. Singh, B.; Pal, L. Radiation crosslinking polymerization of sterculia polysaccharide-PVA-PVP for making hydrogel wound dressings. Int. J. Biol. Macromol. 2011, 48, 501-510. [CrossRef]

31. Göpferich, A. Mechanisms of polymer degradation and erosion. Biomaterials 1996, 17, 103-114. [CrossRef]

32. GD, M. Quantifying wound fluids for the clinician and researcher. Ostomy Wound Manag. 1994, 40, 66-69.

33. Parikh, D.V.; Fink, T.; Delucca, A.J.; Parikh, A.D. Absorption and swelling characteristics of silver (I) antimicrobial wound dressings. Text. Res. J. 2011, 81, 494-503. [CrossRef]

34. Kamoun, E.A.; Kenawy, E.S.; Chen, X. A review on polymeric hydrogel membranes for wound dressing applications: PVA-based hydrogel dressings. J. Adv. Res. 2017, 8, 217-233. [CrossRef]

35. Shi, S.; Peng, X.; Liu, T.; Chen, Y.N.; He, C.; Wang, H. Facile preparation of hydrogen-bonded supramolecular polyvinyl alcohol-glycerol gels with excellent thermoplasticity and mechanical properties. Polymer 2017, 111, 168-176. [CrossRef]

36. Wang, R.-M.; Zheng, S.-R.; Zheng, Y.-P. Matrix materials. In Polymer Matrix Composites and Technology; Elsevier: Amsterdam, The Netherlands, 2011; pp. 101-548.

37. Sun, B.; Tian, Y.; Chen, L.; Jin, Z. Food Hydrocolloids Linear dextrin as curcumin delivery system: Effect of degree of polymerization on the functional stability of curcumin. Food Hydrocoll. 2018, 77, 911-920. [CrossRef]

38. Campus, K.; Kanchanaburi, M.L.; Road, S. Nanocomposites Based on Cassava Starch and Chitosan-Modified Clay: Physico-Mechanical Properties and Biodegradability in Simulated Compost Soil. J. Braz. Chem. Soc. 2017, 28, 649-658.

39. Aghazadeh, M.; Karim, R.; Abdul Rahman, R.; Sultan, M.T.; Johnson, S.K.; Paykary, M. Effect of Glycerol on the Physicochemical Properties of Cereal Starch Films. Food Technol. Econ. Eng. Phys. Prop. 2018, 36, 403-409. [CrossRef]

40. Shrotriya, S.; Ranpise, N.; Satpute, P.; Vidhate, B. Skin targeting of curcumin solid lipid nanoparticles-engrossed topical gel for the treatment of pigmentation and irritant contact dermatitis. Artif. Cells Nanomed. Biotechnol. 2018, 46, 1471-1482. [CrossRef]

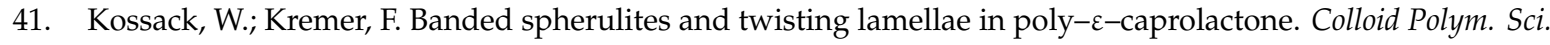
2019, 297, 771-779. [CrossRef]

42. Verhoeven, N.; Neoh, T.L.; Furuta, T.; Yamamoto, C.; Ohashi, T.; Yoshii, H. Characteristics of dehydration kinetics of dihydrate trehalose to its anhydrous form in ethanol by DSC. Food Chem. 2012, 132, 1638-1643. [CrossRef] 
43. Bueno, C.Z.; Moraes, Â.M. Development of Porous Lamellar Chitosa-Alginate Membranes: Effect of Different Surfactants on Biomaterial Properties. J. Appl. Polym. Sci. 2011, 122, 624-631. [CrossRef]

44. Del Prado-Audelo, M.L.; Magaña, J.J.; Mejía-Contreras, B.A.; Borbolla-Jiménez, F.V.; Giraldo-Gomez, D.M.; Piña-Barba, M.C.; Quintanar-Guerrero, D.; Leyva-Gómez, G. In vitro cell uptake evaluation of curcumin-loaded PCL/F68 nanoparticles for potential application in neuronal diseases. J. Drug Deliv. Sci. Technol. 2019, 52, 905-914. [CrossRef]

45. Blattner, C.M.; Coman, G.; Blickenstaff, N.R.; Maibach, H.I. Percutaneous absorption of water in skin: A review. Rev. Environ. Health 2014, 29, 175-180. [CrossRef]

46. Tagami, H.; Kanamaru, Y.; Inoue, K.; Suehisa, S.; Iwatsuki, K.; Yoshikuni, K.; Yamada, M. Water sorption-desorption test of the skin in vivo for functional assessment of the stratum corneum. J. Invest. Dermatol. 1982, 78, 425-428. [CrossRef]

47. Van der Molen, R.; Spies, F.; van’t Noordende, J.M.; Boelsma, E.; Mommaas, A.M.; Koerten, H.K. Tape stripping of human stratum corneum yields cell layers that originate from various depths because of furrows in the skin. Arch. Dermatol. Res. 1997, 289, 514-518. [CrossRef]

48. Kucukturmen, B.; Oz, U.C.; Bozkir, A. In Situ Hydrogel Formulation for Intra-Articular Application of Diclofenac Sodium-Loaded Polymeric Nanoparticles. Turk. J. Pharm. Sci. 2017, 14, 56-64. [CrossRef]

49. Zhang, N.; Said, A.; Wischke, C.; Kral, V.; Brodwolf, R.; Volz, P.; Boreham, A.; Gerecke, C.; Li, W.; Neffe, A.T.; et al. Poly[acrylonitrile-co-(N-vinyl pyrrolidone)] nanoparticles-Composition-dependent skin penetration enhancement of a dye probe and biocompatibility. Eur. J. Pharm. Biopharm. 2017, 116, 66-75. [CrossRef]

50. Goto, N.; Morita, Y.; Terada, K. Deposits from Creams Containing 20\% (w/w) Urea and Suppression of Crystallization (Part 2): Novel Analytical Methods of Urea Accumulated in the Stratum Corneum by Tape stripping and Colorimetry. Chem. Pharm. Bull. 2016, 64, 1092-1098. [CrossRef]

51. Langer, R. New Methods of Drug Delivery. Science 1990, 249, 1527-1533. [CrossRef]

52. Urrejola, M.C.; Soto, L.V.; Zumarán, C.; Peñaloza, P.; Álvarez, B.; Fuentevilla, I.; Haidar, Z.S. Sistemas de Nanopartículas Poliméricas II: Estructura, Métodos de Elaboración, Características, Propiedades, Biofuncionalización y Tecnologías de Auto-Ensamblaje Capa por Capa (Layer-by-Layer Self-Assembly). Int. J. Morphol. 2018, 36, 1463-1471. [CrossRef]

53. Lademann, J.; Richter, H.; Teichmann, A.; Otberg, N.; Blume-Peytavi, U.; Luengo, J.; Weiß, B.; Schaefer, U.F.; Lehr, C.M.; Wepf, R.; et al. Nanoparticles-An efficient carrier for drug delivery into the hair follicles. Eur. J. Pharm. Biopharm. 2007, 66, 159-164. [CrossRef] 